\title{
Pour des compétences professionnelles des enseignant-e-s intégrant le genre
}

\section{Sigolène Couchot-Schiex}

\section{(2) OpenEdition}

\section{Journals}

Édition électronique

URL : http://journals.openedition.org/trema/3584

DOI : $10.4000 /$ trema.3584

ISSN : 2107-0997

Éditeur

Faculté d'Éducation de l'université de Montpellier

Édition imprimée

Date de publication : 1 décembre 2016

Pagination : $29-40$

ISBN : 979-10-96627-02-8

ISSN : 1167-315X

Référence électronique

Sigolène Couchot-Schiex, « Pour des compétences professionnelles des enseignant-e-s intégrant le genre », Tréma [En ligne], 46 | 2016, mis en ligne le 01 avril 2017, consulté le 19 avril 2019. URL : http:// journals.openedition.org/trema/3584 ; DOI : 10.4000/trema.3584

Ce document a été généré automatiquement le 19 avril 2019

Trema 


\title{
Pour des compétences professionnelles des enseignant-e-s intégrant le genre
}

\author{
Sigolène Couchot-Schiex
}

1 C'est bien l'égalité, entendue comme valeur promouvant l'égal accès aux droits pour les femmes et les hommes, qui a été retenue par les institutions françaises pour fonder l'esprit de la République. Toutefois, si l'égalité des sexes est une valeur communément admise voire bien-pensante, le genre est un concept qui la dépasse en intégrant le questionnement collectif des rapports sociaux entre les sexes, dans les rapports de domination/subordination qu'ils exercent sur les individus. Le genre est l'opérateur d'un système (Connell, 1987 ; Messner, 1990 ; Parini, 2006 ; Pétrovic, 2014) qui relaie et diffuse les informations que les individus s'approprient (ou non) concernant les manières d'être un homme ou d'être une femme, de faire l'homme ou de faire la femme qui s'érigent en normes sociales dans un contexte donné. Restreindre les questions sur le genre à l'égalité des sexes fait courir le risque d'orienter les réflexions et les actions qui en découlent à contre-sens des orientations portées par les études scientifiques sur le genre en évacuant la visée systémique et transformative.

2 Le genre constitue le concept clé de la compréhension du social, dépassant la distinction du sexe biologique et du sexe social, il amène à considérer que les comportements sexués sont des modèles construits culturellement et historiquement (Goffman, 2002; Blinde et Taub, 1992). Porter la réflexion sur le genre, nécessite une déconstruction des a priori et des clichés communs, ce qui n'est guère immédiatement accessible, ni dans le cadre de l'exercice citoyen ni dans le cadre de l'exercice professionnel d'enseignement, d'éducation ou de formation. C'est dans cet espace professionnel que s'exercera notre réflexion.

3 La nouvelle loi d'orientation et de programmation de 2013 dite de Refondation de l'école de la République (9 juillet 2013) précise les gestes professionnels attendus, et inscrit parmi ses orientations, les questions liées à l'égalité des sexes et des sexualités en formation initiale comme en formation continue pour l'ensemble des personnels dans les 
équipes éducatives. Si nous persistons dans la voie de parler des questions de genre plutôt que d'égalité, comment ce questionnement peut-il s'intégrer aux compétences professionnelles? La notion de compétence souffre d'un flou sémantique, mais on peut retenir de prime abord qu'elle est apprise et inférée aux situations professionnelles (Fernagu Oudet, 2008). On peut retenir également qu'elle se situe dans l'action réussie. La littérature fournit assez rarement l'occasion de décrire les processus par lesquels les compétences se développent et transforment les pratiques professionnelles. C'est pourquoi nous souhaitons, dans cet article poser la question de la possibilité d'intégrer les enjeux du genre dans le processus de développement des compétences professionnelles des enseignant-e-s ? À quelles conditions cela peut-il être possible ? Pour quels bénéfices escomptés?

\section{De l'égalité au genre}

4 L'égalité des sexes est une valeur largement partagée dont nombre de pays tentent d'accentuer la mise en œuvre depuis la fin du XXe siècle, comme cela a été le cas dès 1957 par le traité de Rome au sein de la Communauté Économique Européenne (CEE) qui adopte le principe d'une rémunération égale pour un travail de valeur égale, puis en 1998, par l'affirmation que l'égalité des sexes se devait, dès lors, d'être prise en compte dans toutes les politiques, dans toutes les instances publiques et privées et à tous les niveaux au sein de l'Union Européenne (UE). Pour atteindre les objectifs visés, l'UE s'est appuyée sur le concept de gender mainstreaming. Ce « concept-méthode » de l'approche intégrée de l'égalité (Dauphin et Sénac-Slawinski, 2008), est mis en avant pour favoriser le passage de l'égalité déclarée (de jure) à l'égalité dans les faits (de facto), dans la réalité ordinaire et quotidienne depuis les années 2000.

5 Le bilan de ce volontarisme et de ces actions est quelque peu pessimiste dans ses visées démocratiques. Bilan pessimiste d'un point de vue politique: "La dialectique entre singularité et similarité enferme les non-frères dans une singularisation incompatible avec un processus démocratique d'égalité comme une relation de non-domination structurelle » (Sénac, 2015, page 80). L'égalité de droit n'empêche pas la production des inégalités de fait qui engendrent une exclusion structurelle des dominés (dont les femmes). Hommes et femmes sont considérés comme égaux en droit, donc similaires, tout en étant différents dans leur essence, donc singuliers. Sénac conclut en posant le dilemme de la construction d'un monde égalitaire selon deux alternatives : la déconstruction des inégalités, ou la pensée d'une alternative qui renouvelle les cadres déterministes des rapports sociaux (Sénac, 2015, page 198). Cette dernière alternative conduirait à un débat social et politique radical. Bilan pessimiste d'un point de vue de l'application des principes d'égalité dans le système éducatif : trente années de travaux scientifiques et de promulgation de lois «accusent le système éducatif, malgré les principes d'égalité qui l'anime, de discriminer les filles et les femmes" (Mosconi, 1989; Duru-Bellat, 1990; Baudelot-Establet, 1992 ; Bourdieu, 1998 ; Cacouault-Bitaud et Gaussot, 2012 ; cité-e-s par Collet, 2012).

6 La conception scientifique française sur le genre heurte l'imaginaire défendu par certains groupes de «l'égalité dans la différence » (Cacouault-Bitaud et Gaussot, 2012, p. 86). Cet idéal rejoint le dilemme posé par Sénac entre singularité et similarité. Puisque l'on dispose depuis plus de trente ans de données convergentes qui démontrent que cette position ne permet pas de déstabiliser les conditions de fabrication des inégalités sociales, 
ne doit-on pas oser penser les problèmes autrement? Alors osons les penser à partir du genre. Osons étudier cette problématique éducative qui se situe au carrefour des questions politiques et scientifiques, au service d'une pensée alternative en éducation dans la didactique professionnelle et les parcours de formation des enseignant-e-s.

\section{Des compétences professionnelles sur le genre?}

7 L'acquisition, la construction de cette compétence dans l'espace professionnel est désormais légitimée par les textes de la réforme de la formation des enseignants ( 9 juillet 2013) par la présence dans les prescriptions de possibilités d'accéder à ces questions, par le biais de l'égalité. Au-delà de l'inscription thématique dans la formation, les conditions de la formation par l'alternance intégrative partageant l'année de stage entre temps de formation et exercice du métier d'enseignant-e dans une classe, devraient constituer un terrain favorable pour la conquête de cette compétence sur le genre chez les enseignante-s stagiaires en formation initiale, puisqu'elle rend possible des allers retours entre étayage théorique et retour réflexif sur la pratique (Schön, 1994). Évidemment l'impact des conditions d'organisation ne doit pas être surévalué dans ce processus d'acquisition et de développement qui se doit avant tout d'être attentif à la personne, aux « aspects humains, affectifs et cognitifs » (Fernagu Oudet, 2008, p. 211), engageant à la fois la personne mais aussi le collectif dans lequel il s'insère ou souhaite s'insérer.

Perrenoud (1997) présente les compétences professionnelles comme «des capacités à mobiliser diverses ressources cognitives pour faire face à des situations singulières ». Cela engage à considérer d'une part que les compétences mobilisent des savoirs, savoir-faire ou attitudes mais n'y sont pas réduites, d'autre part, que cette mobilisation n'est pertinente que confrontée à une situation nécessairement singulière, unique, non reproductible à l'identique, comme ici, dans le contexte de l'enseignement. Ainsi, une compétence ne s'enseigne pas, ne se transmet pas, elle s'acquiert voire elle se conquiert, se développe. Dans le référentiel des compétences professionnelles des métiers du professorat et de l'éducation (1-7-2013), les compétences sont limitées à des réflexions générales sur le vivre ensemble et le respect mutuel, ou bien attirent l'attention sur la reconnaissance des stéréotypes et des préjugés. Mais rien n'est dit de ce qu'il faut faire de ce repérage, ni en quoi il pourrait servir d'appui à des transformations des préjugés. Il reste cependant possible de dépasser ces restrictions pour intégrer des réflexions sur le genre en didactique des disciplines, comme certaines le proposent déjà (l'histoire ou l'éducation physique et sportive (EPS)). Les paramètres qui participent au cours de l'activité d'enseignement-apprentissage à la fabrication des inégalités sexuées en classe, sont maintenant bien identifiés, à partir des travaux scientifiques menés depuis les années 2000. Les résultats des études réalisées montrent que ces écarts sont accentués par la nature du curriculum (choix des contenus d'enseignement et pratiques de référence), les interactions enseignant-e/élèves, la nature des régulations ainsi que les attentes dont sont porteurs les enseignant-e-s à l'égard des élèves. Il est désormais acquis que c'est tout un curriculum caché, différencié selon le sexe des élèves qui est ainsi transmis à l'école à travers les enseignements eux-mêmes.

9 La construction de compétences professionnelles fait l'objet d'une production scientifique depuis les années 1990 avec l'avènement de la didactique professionnelle qui se propose d'analyser "les situations de travail afin de mieux concevoir et organiser la formation " (Rey, 2014, p. 36) et dans l'enseignement (Vinatier, 2009). Ces travaux ont permis de 
repérer des invariants de l'acquisition de la compétence qui ont été modélisés sous forme de schèmes en référence aux travaux piagétiens. D'après Pastré (Pastré, Mayen et Vergnaud, 2006) l'acteur-trice utilise toujours les mêmes concepts et théorèmes pour identifier toute situation appartenant à un même type mais l'activité déployée s'ajuste à la situation selon une démarche adaptative.

Directement concernée par la gestion des différences qui apparaissent de manière cruciale au cours des séances scolaires, tant les filles et les garçons dissemblables, l'EPS s'interroge sur le rapport au corps, à la performance, au rapport à l'autre par la médiation des pratiques physiques sportives, culturelles et sociales. C'est dans ce contexte spécifique que s'illustrera la démarche adaptative envisagée dans cette étude.

\section{Vers l'acquisition des compétences professionnelles sur le genre.}

11 Un exemple tiré de la formation des enseignant-e-s novices en EPS (Couchot-Schiex, Coltice et Cogérino, 2009) nous permettra d'identifier le cheminement de la construction de la compétence sur le genre en le confrontant aux routines définies par Pastré et al. (2006) et en nous appuyant sur des indicateurs du modèle CompAS proposé par Parmentier et Paquay (2002). Ces auteurs précisent que cet outil vise à analyser les situations d'enseignement/apprentissage en vue de favoriser la construction des compétences par les apprenants dans une tentative de prise en compte de la complexité des situations tant du coté de l'enseignement que du coté de l'apprentissage.

\section{III.1. Un exemple en EPS.}

12 La situation de formation qui supporte l'analyse est issue de la formation d'enseignant-e-s novices du secondaire (collège et lycée) en Éducation Physique et Sportive. Pendant une année, un groupe a bénéficié d'une sensibilisation à la question de la mixité et a été informé des résultats de recherches antérieures dans la discipline et de questions plus théoriques sur le genre. Parmi eux, quatre enseignant-e-s novices volontaires ont été observé-e-s in situ au cours de 2 séances d'EPS avec leurs classes habituelles. Des vidéos de chaque séance observée ont été réalisées, suivies par un entretien d'autoconfrontation ciblant des moments où « l'apprendre ensemble » filles et garçons pouvait être considéré comme crucial.

13 En fin d'année, leur participation à un entretien semi-directif a permis d'approfondir le vécu personnel et professionnel de ces quatre stagiaires et un court écrit sous forme de bilan proposé à l'ensemble du groupe. Les résultats montrent que cette compétence n'est pas aisée à conquérir, même lorsque l'enseignant-e stagiaire fait part d'un volontarisme à s'emparer de ce questionnement.

Les résultats peuvent être lus à partir du modèle CompAS (Parmentier et Paquay, 2002) à propos des compétences professionnelles.

Le premier indicateur retenu consiste à « identifier le problème posé ». Pour l'ensemble du groupe (15 enseignant-e-s stagiaires), les apports de connaissance montrent peu d'efficacité, puisque pour les deux tiers seulement, une certaine mobilisation dans la pratique professionnelle est rapportée dans le court écrit proposé à toute-s en fin d'année. C'est en effet, une étape cruciale qui doit pouvoir dépasser les allants-de-soi et le 
sens commun dans les rapports de sexe, ce qui constitue une première difficulté dont témoignent certains retours des étudiant-e-s $(5 / 15)$ « je n'ai rien à dire à ce sujet », «je n'ai pas rencontré de problème» ou encore «la gestion de la mixité n'est pas ma préoccupation prioritaire ». Pour les autres (10/15) les premières expériences d'enseignement sont l'occasion d'observer des comportements différenciés des filles et des garçons en EPS où la qualité motrice et la socialisation sportive accentuent les différences. Les prises en compte restent d'ailleurs collées aux stéréotypes de sexe, à la perception générale de l'activité des élèves en attribuant des comportements à chaque catégorie de sexe : les filles sont...font, les garçons sont...font.

Le second indicateur présenté dans le modèle CompAS est celui de «l'organisation des activités d'apprentissage ». Dans notre exemple, le groupe a bénéficié d'aide à la préparation de séquences et de séances d'enseignement. L'entrée dans la séquence et l'évaluation ont en particulier été travaillées en posant la question de la connotation de l'activité physique support de l'enseignement (connotation dite féminine, masculine ou indifférenciée) pouvant activer plus ou moins fortement les stéréotypes et les préjugés sexués et de l'organisation des élèves (modes de groupement). Cette préparation accompagnée semble avoir activé une vigilance sur certains points: «En lutte, j'ai organisé des surfaces de combat en séparant les filles des garçons [...]. Je me suis surprise à avoir créé cette séparation [...]. J'ai donc rétabli en ne départageant pas [...]». Il est difficile de conclure que la compétence est en voie d'acquisition, mais elle semble déjà permettre de repérer des situations du temps didactique dans les-quelles la réflexion peut s'engager, porteuse de premières modifications des pratiques enseignantes. Lorsque l'enseignant-e novice dit considérer les questions liées au genre, il s'agit en fait essentiellement de la gestion de la mixité dans la classe. En outre, cela concerne davantage la phase de conception des enseignements ou l'évaluation (Coltice et CouchotSchiex, 2006), mais plus rarement les situations d'apprentissage ou les régulations pendant la séance.

17 Le troisième indicateur du modèle CompAS concerne «L'exploitation des ressources y compris les acquis antérieurs». Des données ont pu être recueillies au cours de l'entretien de fin d'année. Certain-e-s enseignant-e-s novices ont à cette occasion, parlé de leur propre intérêt pour les questions de genre (ou désintérêt) et montré comment leur lecture des situations d'enseignement avait pu évoluer au cours de l'année à partir de quelques indicateurs qu'ils ont pu identifier. Souvent un «événement marquant » a pu être à l'origine d'une prise de conscience, en situation, des effets des choix ou des actions enseignantes. Dans ce cas, la déstabilisation conduit à envisager la situation autrement et à initier quelques modifications des actions enseignantes pour envisager une réponse qui semble plus pertinente.

18 Le quatrième indicateur : « agir/interagir » permet quelques repérages de la construction de la compétence dans la situation en train de se réaliser, dans notre exemple à partir de l'observation des séances des quatre enseignant-e-s novices volontaires et des entretiens d'auto-confrontation. Ces observations donnent l'occasion de repérer la compétence dans l'action. Des comportements des enseignant-e-s en action, il transparait de leurs décisions didactiques et pédagogiques, de leurs attitudes professionnelles, une réelle difficulté au moment de l'action elle-même à prendre conscience de son enjeu du point de vue du genre. Puis au cours de l'entretien d'auto-confrontation de la difficulté à expliciter les décisions et plus encore à percevoir les implications qu'elles engendrent sur l'apprentissage ou la gestion des élèves au sein de la classe mixte. 
19 Ici, l'accompagnement par le/la formateur/trice s'est avéré essentiel. En effet, c'est lui/ elle qui sélectionne l'extrait de la situation de classe auquel il/elle confronte ensuite l'enseignante à l'issue de la séance. Ce faisant, il/elle aide d'une part à identifier le moment crucial et participe par son questionnement à construire le problème. On peut dire que la compétence à lire les situations avec le genre s'apparente à une compétence tacite (Pastré, 1999) ou implicite, davantage qu'à une compétence explicite. Les enseignant-e-s novices ont du mal à s'exprimer à ce sujet, à percevoir les effets de leurs actions mises en œuvre dans l'urgence de la situation. Cette compétence s'avère souvent masquée par d'autres préoccupations comme celle de la mise en activité des élèves telles que nous avons pu le recueillir au cours des écrits de fin d'année et des entretiens d'autoconfrontation.

Les indicateurs «réfléchir, évaluer» et «structurer, intégrer» sont susceptibles de contribuer à construire du sens et à faciliter l'accès à la transférabilité de la compétence visée. Au cœur de chaque situation d'enseignement-apprentissage, l'enseignant-e par ses actions, ses décisions, les dispositifs et modalités d'apprentissage qu'il-elle met en œuvre génère des conséquences, des effets sur les apprentissages des filles et des garçons dans la classe mixte.

21 Au terme de cette lecture des compétences sur le genre outillée par la grille CompAs, nous pouvons retenir trois conditions qui marquent l'accès ou le développement des compétences recherchées.

Tout d'abord, si le volontarisme de l'enseignant-e est une condition favorable, elle n'est pas suffisante à la construction de la compétence sur le genre. Le retour réfléchi sur l'action, susceptible de permettre de décrypter les effets favorables ou défavorables des décisions et des actions pendant la séance sur les apprentissages des élèves est difficile. Il nécessite de passer d'une position personnelle concernant la vision sexuée du monde que porte chaque enseignant-e y compris dans les situations professionnelles desquelles il/ elle est acteur/actrice, à une position professionnelle.

Ensuite, l'accompagnement par des formateurs-trices à certains moments clés est bénéfique au développement des compétences. Cela pointe l'intérêt et l'importance du cadre de la formation. Si le retour réflexif est nécessaire, le cadre de formation qui rend possible son engagement, au sein d'un collectif semble fructueux.

24 Enfin, dans le cadre professionnel, il est indispensable que les enjeux du genre entrent en congruence avec les valeurs et orientations générales portées par l'enseignant-e. Ces valeurs sont issues des acquis et des ressources antérieures de l'enseignant-e en tant que sujet et sont intégrées à sa subjectivité. Un travail sur les valeurs, dans l'exercice professionnel, semble donc nécessaire afin de faciliter les mises en lien avec les propositions d'actions enseignantes de manière à pouvoir les intégrer dans des perspectives appartenant au périmètre du référentiel de compétences des professeurs et des éducateurs.

\section{III.2. Des conditions favorables?}

Dans l'exemple présenté, les enseignant-e-s novices renvoient les situations d'enseignement-apprentissage à un indicateur de l'activité déployée par les élèves qui sert de théorème de référence. L'activité adaptative peut les amener à modifier la composition des groupes de travail, le but ou la modalité de la situation proposée pour 
que les élèves restent engagés dans le travail. Dans ce sens, on peut affirmer que le concept de genre, ne sert pas la lecture des situations didactiques auxquelles ils/elles sont confronté-e-s. Mais alors, comment peut-on engager les enseignant-e-s dans l'acquisition de cette compétence ? À partir de propositions antérieures (Samurcay et Pastré, 1995 ; Rey, 2014) nous avons identifié quatre points clés susceptibles de conduire ce processus d'acquisition.

- L'acteur-trice : l'enseignant-e ou l'éducateur-trice peut s'interroger sur ce qu'il-elle vit en tant qu'individu ou professionnelle en le connectant à un questionnement sur le genre. Les formations engagent le plus souvent à cette confrontation personnelle. Lire une situation en intégrant les enjeux dont est porteur le genre, invite à se questionner sur soi-même et le rapport que chacun-e entretient avec le monde.

27 - La mise en activité de problématisation : à partir de l'exposé d'une situation constituée comme une étude de cas, il s'agit de permettre, par des tâtonnements successifs, individuellement, par petits groupes ou avec un-e expert-e de ces questions, de mettre en rapport les données de la situation avec les savoirs théoriques ou des modèles explicatifs présentant des indicateurs, des critères repérables. Cette mise en activité doit permettre de retrouver les facteurs scientifiquement identifiés.

28 - L'expérience de la situation : c'est l'étape de la confrontation au réel, à la décision dans l'urgence. À partir de situations de classe expérimentées ou observées, il s'agit de permettre le va et vient entre les savoirs et les choix à opérer en temps réel. Cette confrontation à l'expérience nécessite un accompagnement réflexif, un guidage, un outillage pour que se développe progressivement la compétence.

29 - Le pilotage par les valeurs : les valeurs portées par l'enseignant-e sont au fondement des actions qu'il-elle met en œuvre. Ainsi l'orientation par les valeurs, pilote les choix effectués, qu'ils soient déterminés dans l'urgence de la situation ou dans la tranquillité de la conception.

30 Les éléments consignés dans le tableau ci-dessous récapitulent les conditions susceptibles de favoriser l'acquisition d'une compétence professionnelle sur le genre par les enseignant-e-s. 
Tableau 1 : Points clés de la formation pour la construction d'une compétence sur le genre

\begin{tabular}{|c|c|c|c|c|}
\hline $\begin{array}{l}\text { Construction de la } \\
\text { compétence }\end{array}$ & Acteur & Problématisation & Situation & Valeur \\
\hline $\begin{array}{c}\text { Critères du } \\
\text { modèle théorique }\end{array}$ & $\begin{array}{l}\text { Engagement } \\
\text { personnel et } \\
\text { professionnel }\end{array}$ & $\begin{array}{l}\text { Mise en situation } \\
\text { de repérage des } \\
\text { indicateurs et } \\
\text { explicitation par } \\
\text { les savoirs }\end{array}$ & $\begin{array}{c}\text { Expérience } \\
\text { vécue par la } \\
\text { confrontation } \\
\text { au réel } \\
\text { Accompagne- } \\
\text { ment réflexif }\end{array}$ & $\begin{array}{l}\text { Fondement des } \\
\text { actions réalisées } \\
\text { dans l'urgence } \\
\text { ou non }\end{array}$ \\
\hline $\begin{array}{l}\text { Eléments tirés de } \\
\text { l'exemple }\end{array}$ & $\begin{array}{c}\text { Volontarisme } \\
\text { antérieur } \\
\text { personnel } \\
+ \\
\text { Information a } \\
\text { minima des } \\
\text { enseignant-e-s } \\
\text { sur les questions } \\
\text { que pose le } \\
\text { genre dans } \\
\text { l'espace d'éduca- } \\
\text { tion et d'ensei- } \\
\text { gnement }\end{array}$ & $\begin{array}{l}\text { Construction } \\
\text { accompagnée de } \\
\text { la problématisa- } \\
\text { tion pour l'ensei- } \\
\text { gnement (cadre } \\
\text { didactique et/ou } \\
\text { transversal) } \\
\text { Étayage } \\
\text { scienti-fique }\end{array}$ & $\begin{array}{c}\text { Analyse de la } \\
\text { pratique profes- } \\
\text { sionnelle dans } \\
\text { des espaces de } \\
\text { formation dédiés : } \\
\text { analyse de } \\
\text { pratique, } \\
\text { initiation à la } \\
\text { recherche, } \\
\text { projets indivi- } \\
\text { duels ou } \\
\text { collectifs }\end{array}$ & $\begin{array}{l}\text { Enjeux du } \\
\text { questionnement } \\
\text { par le genre. } \\
\text { Transformations } \\
\text { personnelles et } \\
\text { professionnelles. } \\
\text { Effets sur la } \\
\text { conception et la } \\
\text { régulation des } \\
\text { situations } \\
\text { éducatives et } \\
\text { didactiques }\end{array}$ \\
\hline
\end{tabular}

Tableau I: Points clés de la formation pour la construction d'une compétence sur le genre construit de manière non linéaire mais plutôt spiralaire, au fur et à mesure de l'avancée dans le développement de la compétence. L'ambition de la formation est celle de la transformation des pratiques enseignantes. Celle-ci n'est cependant pas dissociable d'une transformation de la personne, car les questions de genre touchent aux croyances personnelles qui fondent les valeurs de l'individu. Faire réfléchir sur les pratiques professionnelles d'enseignement engendre une réflexion sur soi, sur la manière d'envisager les relations sociales entre les sexes, dans la classe et aussi dans la société dans son ensemble. Il s'agit d'engager une participation active au processus de transformation professionnelle. Transformer ses pratiques, c'est se transformer pour transformer les élèves dans leur rapport au monde, incluant le rapport aux savoirs et aux autres.

\section{Conclusion}

Acquérir et développer une compétence professionnelle à enseigner avec le genre n'est pas un leurre. Formatrice depuis dix ans, l'occasion de recueillir des témoignages de certain-e-s enseignant-e-s novices jalonne notre parcours. Outre l'intérêt qu'ils-elles ont eu à réfléchir aux enjeux que propose le genre et expérimenter des outils pour les apprentissages ou la gestion de la classe mixte, ils/elles racontent que cette approche genrée modifie leur regard sur l'enseignement mais aussi sur la vie sociale. Ils/elles acquièrent une compétence mobilisable pour l'enseignement transférable à d'autres espaces y compris en dehors de la sphère professionnelle. Quelles sont les conditions qui permettraient de garantir des espaces de formation favorables à ce développement ? Tout d'abord, il est important de reconnaitre la place de la formation. Tous les enseignant-e-s 
novices bénéficient d'un temps de formation à l'issue de la réussite aux concours d'enseignement; il est important de s'en saisir pleinement en utilisant au mieux les conditions actuelles de la formation des enseignant-e-s, notamment la formation en alternance. Celle-ci rend possible l'organisation de dispositifs utiles au développement des compétences, avec un accompagnement par des formateurs-trices mais aussi par des pairs via des groupes d'analyse de pratique et de mutualisation des ressources (supports) adaptées pour l'enseignement. Les ressources et les espaces préexistants sont déjà nombreux bien qu'encore insuffisamment diffusés.

Le travail en équipe des formateurs-trices peut être le lieu privilégié de débats pour réguler et évaluer les effets de la formation. Les formateurs-trices peuvent aussi se former aux compétences professionnelles intégrant les questions sur le genre! Quand une équipe dispose des compétences, il faut veiller à mettre en place les conditions de leur diffusion au moins à l'échelon local! Ces formations peuvent être centrées à la fois sur la compétence à enseigner dans la didactique d'une discipline et sur la compétence à éduquer au respect mutuel ou à la lutte contre les préjugés. Il s'agit de prendre en charge à la fois le "vivre ensemble » et l' " apprendre ensemble » dans les disciplines scolaires (Couchot-Schiex, 2012 ; 2014 ; Dhume, 2016).

Le genre met en évidence les paradoxes de l'institution: il faut avancer sur les problématiques socialement vives... mais il reste difficile d'en débattre ouvertement. Si certains acteurs ou certaines actrices de l'éducation et de la formation se mobilisent depuis plusieurs années, tentant de poser ce problème dans l'espace éducatif, ils-elles sont plutôt perçu-e-s comme des perturbateurs-trices du fonctionnement ordinaire de l'école. Pourtant, le genre participe des " problèmes publics » (Cefaï et Terzi, 2012) qui se posent et se recomposent de manière spécifique dans l'espace scolaire (OUIEP, 2016), et à ce titre mérite d'être considéré à la hauteur de ses enjeux politiques pour la société de demain.

\section{Annexe}

LOI n²014-873 du 4 août 2014 pour l'égalité réelle entre les femmes et les hommes (1)

\section{Extrait}

Article 1

L'État et les collectivités territoriales, ainsi que leurs établissements publics, mettent en œuvre une politique pour l'égalité entre les femmes et les hommes selon une approche intégrée. Ils veillent à l'évaluation de l'ensemble de leurs actions.

La politique pour l'égalité entre les femmes et les hommes comporte notamment :

1. Des actions de prévention et de protection permettant de lutter contre les violences faites aux femmes et les atteintes à leur dignité ;

2. Des actions visant à renforcer la lutte contre le système prostitutionnel ;

3. Des actions destinées à prévenir et à lutter contre les stéréotypes sexistes ;

4. Des actions visant à assurer aux femmes la maîtrise de leur sexualité, notamment par l'accès à la contraception et à l'interruption volontaire de grossesse ;

5. Des actions de lutte contre la précarité des femmes ;

6. Des actions visant à garantir l'égalité professionnelle et salariale et la mixité dans les métiers ; 
7. Des actions tendant à favoriser une meilleure articulation des temps de vie et un partage équilibré des responsabilités parentales ;

8. Des actions visant à favoriser l'égal accès des femmes et des hommes aux mandats électoraux et aux fonctions électives, ainsi qu'aux responsabilités professionnelles et sociales ;

9. Des actions visant à garantir l'égalité de traitement entre les femmes et les hommes et leur égal accès à la création et à la production culturelle et artistique, ainsi qu'à la diffusion des œuvres ;

10. Des actions visant à porter à la connaissance du public les recherches françaises et internationales.

\section{BIBLIOGRAPHIE}

Baudelot, C. et Establet, R. (1992). Allez les filles! Paris : Seuil.

Blinde, E. M. et Taub, D. E. (1992). Homophobia and women's sport: the disempowerment of athletes, Sociological focus, 25 (2), 151-166.

Bourdieu, P. (1998). La domination masculine. Paris : Seuil.

Cacouault-Bitaud, M. et Combaz, G. (2012). La formation et le genre, Recherche et Formation, 69.

Cacouault-Bitaud, M. et Gaussot, L. (2012). Autour des mots de la formation « sexe » - " genre », La formation et le genre, Recherche et Formation, 69, 81-94.

Cefaï, D. et Terzi, C. (dir.). (2012). L'expérience des problèmes publics. Perspectives pragmatistes. Paris : EHESS.

Collet, I. (2012). Faux semblants et débats autour du genre et de l'égalité en éducation et formation, Recherche et formation, 70, 121-134.

Coltice, M. et Couchot-Schiex, S. (2006). Regards sur la mixité par un groupe d'enseignants stagiaires en EPS. In G. Cogérino, La mixité en éducation physique. Paroles, réussites, différenciations. Dossier EPS, 67, 122-140.

Connell, R. W. (1987). Gender and Power: Society, the Person and Sexual Politics. Stanford University Press, Stanford, CA.

Couchot-Schiex, S. (2012). Faire apprendre ensemble les filles et les garçons en éducation physique et sportive : une compétence difficile à cerner ? 24e colloque de l'ADMEE-Europe, L'évaluation des compétences en milieu scolaire et professionnel, 11-13 Janvier, Luxembourg.

Couchot-Schiex, S. (2014). Jusqu'où la didactique de l'EPS peut-elle intégrer le genre ? Journée d'études RING et ARGEF, Genre-Enseignement-Egalité, 14 novembre, Paris : MESR.

Couchot-Schiex, S., Coltice, M. et Cogerino, G. (2009). Professeurs stagiaires en EPS face à l'enseignement en mixité. Carrefours de l'éducation, 74, 171-184.

Dauphin, S. et Sénac-Slawinski, R. (2008). Gender mainstreaming : analyse des enjeux d'un 'concept-méthode'. Cahier du genre, 44, 5-16. 
Dhume, F. (2016). Former les enseignant(e)s sur la (non)-discrimination, [en ligne : http:// www.lairedu.fr/media/video/conference/former-enseignantes-non-discrimination/ , consulté le 24 avril 2016.

Duru-Bellat, M. (1990). L'école des filles : quelle formation pour quels rôles sociaux? Paris :

L'Harmattan.

Fernagu Oudet, S. (2008). Organisation du travail et développement de compétences/construire la professionnalisation. Paris : L'Harmattan.

Goffman, E. (2002). L'arrangement des sexes. Paris : La Dispute. Version anglaise 1977.

Messner, M. A. (1990). When bodies are weapons: Masculinity and violence in Sport, International Review for the Sociology of Sport, 25, 203-220.

Mosconi, N. (1989). La mixité dans l'enseignement scolaire : un faux semblant? Paris : PUF.

Parini, L. (2006). Le système de genre : introduction aux concepts et théories. Zurich : Seismo.

Parmentier, P. et Paquay, L. (2002). En quoi les situations d'enseignement-apprentissage favorisent-elles le développement de compétences? Vers un outil d'analyse : le CompAS. Groupe de recherche interdisciplinaire en formation des enseignants et en didactique. Université catholique de Louvain. Document en libre accès. http://www.grifed.ucl.ac.be/CompAS-V3-vd.pdf Perrenoud, P. (1997). De nouvelles compétences professionnelles pour enseigner à l'école primaire. Éducateur, 10, 24-28.

Pastré, P. (1999). La conceptualisation dans l'action : bilan et nouvelles perspectives, Éducation permanente, 139, 13-35.

Pastré, P., Mayen, P. et Vergnaud, G. (2006). La didactique professionnelle. Note de synthèse. Revues Française de Pédagogie, 154, 145-198.

Pétrovic, C. (2014). Le développement de l'identité sexuée au sein du système de genre. In F. Hauwelle, M.-N. Rubio et S. Rayna, L'égalité des filles et des garçons dès la petite enfance. (p. 31- 48). Toulouse : Érès.

Observatoire Universitaire International d'Éducation et Prévention (2016). Penser les nouvelles problématiques éducatives dans une perspective internationale, 16-18 novembre, Bonneuil-sur-Marne : Université Paris-Est Créteil.

Rey, B. (2014). La notion de compétence en éducation et formation : enjeux et problèmes, Bruxelles : De Boeck.

Samurcay, R et Pastré, P. (1995). Outiller les acteurs de la formation pour le développement des compétences, Éducation permanente, 123.

Schön, D. A. (1994). Le praticien réflexif. À la recherche du savoir caché dans l'agir professionnel. Montréal : Éditions Logiques.

Sénac, R. (2015). L'égalité sous conditions. Genre, parité, diversité. Paris : Presses de la Fondation Nationale des sciences politiques.

Vinatier, I. (2009). Pour une didactique professionnelle de l'enseignement, Rennes : PUR.

Arrêté du 27 août 2013 fixant le cadre national des formations dispensées au sein des masters « métiers de l'enseignement, de l'éducation et de la formation » http://www.legifrance.gouv.fr/ affichTexte.do;jsessionid=? cidTexte=JORFTEXT000027905257\&dateTexte=\&oldAction=dernierJOetcategorieLien=id 
Arrêté du 1er juillet 2013 relatif au référentiel des compétences professionnelles des métiers du professorat et de l'éducation http://www.legifrance.gouv.fr/affichTexte.do?

cidTexte=JORFTEXT000027721614\&dateTexte $=\&$ categorieLien $=i d$

\section{RÉSUMÉS}

Si l'égalité est une valeur dont se sont saisies les politiques générales et éducatives, nous proposons dans cet article, de déplacer cette centration en direction des questions portées par le genre et de les intégrer en formation des enseignant-e-s. L'acquisition d'une compétence professionnelle sur le genre est-elle envisageable en formation, à quelles conditions et pour quels enjeux?

Gender equality is a value taken by public and educational policies. In this paper we argue for moving this focus to gender problematics embeded in teacher training. Is a professional competence on gender a conceival proposal? Under what conditions? For what major issue?

\section{INDEX}

Mots-clés : égalité, genre, formation, enseignant-e, compétence

Keywords : equality, gender, teacher training, competence

\section{AUTEUR}

\section{SIGOLÈNE COUCHOT-SCHIEX}

Maîtresse de Conférences - Université Paris-Est Créteil (UPEC), Laboratoire Interdisciplinaire de Recherche sur les Transformations des pratiques Éducatives et des pratiques Sociales (LIRTES) EA 7313, Observatoire Universitaire International Éducation et Prévention (OUIEP) 